\title{
The use of trans-vaginal laparoscopy in ruling out pelvic malignancy: a case report
}

Published online: 14 January 2004

(C) Springer-Verlag Berlin / Heidelberg 2004

\begin{abstract}
Trans-vaginal laparoscopy (TVL) was performed in a patient at high risk for ovarian cancer (OC). During a routine check-up vaginal sonography and color Doppler studies revealed neoangiogenesis with low resistance in both sides. The left ovary was slightly enlarged, and the increased blood vessels were seen in the center and in the periphery of the left ovary. The patient was placed in the lithotomy position after sedation, and a veres needle was inserted in the posterior vaginal vault. A trocar followed, and warm normal saline was injected in the pelvis. The needle was removed, and a telescope was introduced, connected to a light source and a monitor. Excellent visualization was achieved, and the average time of TVL was $30 \mathrm{~min}$. Dilated veins in both adnexa and pelvic tissues due to severe obesity were visualized. TVL helped in establishing the diagnosis, saving this patient from an unnecessary laparoscopy or laparotomy.
\end{abstract}

Keywords Ovarian cancer · Trans-vaginal laparoscopy

\section{Introduction}

The high-risk patients for developing ovarian cancer are postmenopausal women and patients with BOC syndrome [1]. Vaginal sonography accompanied by color Doppler studies and serum Ca125 as a screening measure on asymptomatic patients has failed to reduce OC mortality significantly [2]. Similarly, patients diagnosed with ovarian lesion after imaging most of the times have to undergo unnecessary operations to rule out ovarian cancer (OC). According to the latest reports, for every seven postmenopausal women identified with abnormal ultra-

\footnotetext{
V. Tanos (๘)

Department of Gynecological Oncology,

Evagelistria Medical Center,

P.O. Box 25441, 1309 Nicosia, Cyprus

e-mail: tanosv@spidernet.com.cy

Tel.: +357-22-673340

Fax: +357-22-673305
}

sound (US) and/or elevated Ca125 and undergoing laparoscopy/laparotomy, only one had OC $[1,2,3]$.

The great majority $(>95 \%)$ of women with suspected ovarian cancer are usually postmenopausal women with abnormal pelvic sonography. When a pelvic mass is found, computerized tomography and/or magnetic resonance imaging and serum Ca125 usually follow to help with the diagnosis [4]. However, in more than $70 \%$ of the cases, benign pathology is reported. As a result, many unnecessary laparotomies or laparoscopies are performed in fear of ovarian cancer [3].

Trans-vaginal laparoscopy (TVL) using a 2.8-mmdiameter telescope is a new method that is primarily used to diagnose infertility problems such as endometriosis, pelvic adhesions and other pelvic pathology with great success $[5,6,7]$. TVL was performed on a patient with suspected ovarian/pelvic pathology visualized by ultrasound, suggesting ovarian cancer. It was of a great help in establishing the diagnosis, saving the patient from undergoing a more invasive procedure.

\section{Case report}

The patient was a 60-year-old female, married with two children, who was obese $(110 \mathrm{~kg})$, but generally healthy. She had gained $15 \mathrm{~kg}$ in the last 14 months. During a routine check-up, vaginal sonography and color Doppler studies revealed neoangiogenesis with low resistance on both sides. The left ovary was slightly enlarged, and the increased blood vessels were seen in the center and in the periphery of the left ovary (Fig. 1A). In the right adnexa, increased vascularity with low resistance flow was seen adjacent to the right ovary (Fig. 1D). When TVL was performed varicose veins were visualized in both ovaries (congested ovaries), more prominently in the left ovary (Fig. 1B, C) and in the right fibria (Fig. 1E, F). The warm normal saline used to visualize the pelvis was collected and sent for cytology. Normal cytology was reported. The etiology of these findings seems to be the increased peripheral estrogens due to the increased body mass index. The TVL was uneventful, and the patient was discharged home after $2 \mathrm{~h}$. 
Fig. 1 A patient with bilateral adnexal neoangiogenesis. A Left ovary with low resistance blood flow. B, C Arrows show the varicose veins formed on the left ovary. D Right ovary with low resistance flow on its top, which the TVL eventually shows as the right fibria covered with varicose veins. $\mathbf{E}$ Panoramic view of the right saplings fibria. F Close-up view of the right fibria. Arrows show the distended veins
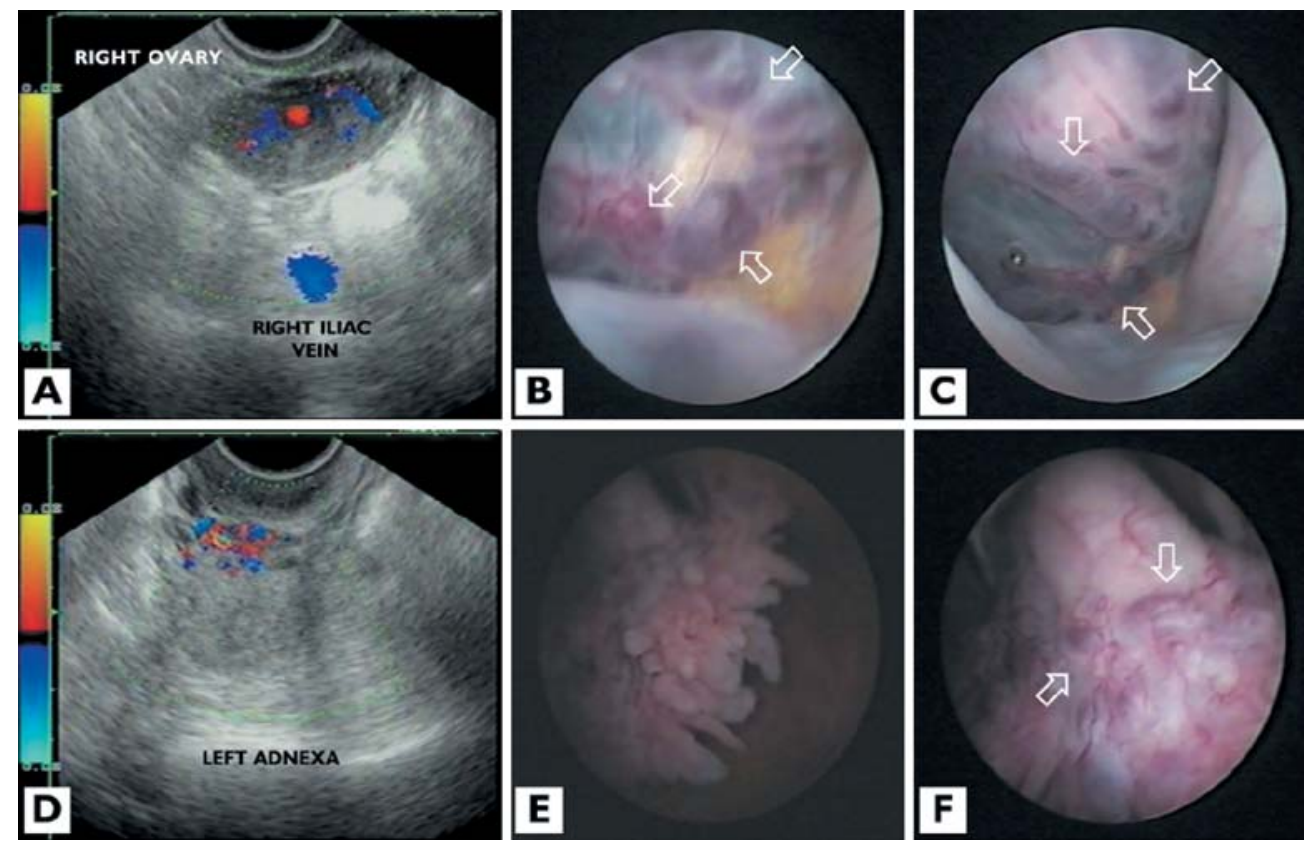

\section{Discussion}

The anatomical position of the ovaries and the delayed onset of symptoms in ovarian cancer are the main reasons for the high mortality rate. The application of vaginal sonography alone or in combination with Ca125 does not seem to reduce the OC mortality rate significantly, while it increases the number of unnecessary laparoscopies/ laparotomies [2, 3]. Hence, the need for a better method of detection of OC at an early stage is necessary. At this stage, the application of an OC detection screening program looks remote. Studies have shown that the application of vaginal sonography and Ca125 serum levels is more efficient for this special group of patients [2], but, again, in order to establish the diagnosis at least with a biopsy implies an invasive procedure with anesthesia with a substantial financial cost and psychological distress for the patient.

Imaging procedures like 3D-ultrasound, color Doppler studies, computerized tomography scanning or magnetic resonance imaging give excellent resolution and are very helpful in identifying an ovarian lesion. However, for the final diagnosis an open biopsy is still necessary. Recently, the fractal dimensions of outlines of sonographically depicted solid components in 160 ovarian tumors were measured using a box-counting method. This study showed that the surface of solid components in cystic epithelial ovarian cancers has a fractal structure and may require different treatment strategies [8]. Of course, there is still the question whether ovarian cancer initiates from the ovarian core or from the surface. The two basic theories generally accepted by the majority of gynecologists worldwide are: (1) the theory of "incessant ovulation," i.e., the switching on and off of cell growth inducing the potential of uncontrolled growth [9] and (2) the theory of "ovulatory age," the more ovulations the greater the risk of developing ovarian cancer [10]. Both theories support the fact that OC initiates from the ovarian cortex surface. The chance to depict ovarian lesions at a very early stage, before even stage I, by imaging techniques has not been tried because it seems unrealistic.

Recently, Leeper et al. reported an increased frequency of occult ovarian carcinomas after prophylactic oophorectomy specimens in high-risk women and concluded that (1) the fallopian tubes and the ovaries should be entirely submitted for histopathological serial sections and (2) that laparoscopy and laparotomy are the surgical modalities of choice to allow inspection of the peritoneal surfaces at the time of prophylactic oophorectomy and collection of fluid for cytologic evaluation [11].

Under these circumstances TVL seems to be an attractive method for examining the adnexae, but also the whole pelvis of women at high risk of OC. In TVL the tissues inspected are floating in normal saline, providing better and more accurate visualization since pelvic lesions can be demonstrated that could not be identified by laparoscopy or laparotomy. Brosens et al. in 2001 reported the diagnosis of micro, filmy adhesions and endometriosis foci within the fallopian tubes and the ovaries that could not be diagnosed by laparoscopy [5]

The problem of spreading the disease after biopsy of a lesion suspected of OC during TVL is of primary importance. However, frozen section or a switch to laparotomy in these cases can be an option. In this case report we can conclude that the method of trans-vaginal laparoscopy enabled direct visualization of the ovaries and pelvis, whereby the cytology obtained excluded pelvic/ovarian malignancies. 


\section{References}

1. DiSaia PJ, Creasman WT (2002) Epithelial ovarian cancer. In: DiSaia PJ, Creasman WT (eds) Clinical gynecologic oncology. Mosby, St Louis, p 289

2. Jacobs IJ, Skates SJ, MacDonald N, et al (1998) Screening for ovarian cancer: A pilot randomized control trial. Lancet 353:1207

3. Rosenthal A, Jacobs I (1998) Ovarian cancer screening. Sem Oncol 25:315

4. Jacobs I, Davies AP, Bridges J, et al (1993) Prevalence screening for ovarian cancer in postmenopausal women by CA125 measurement and ultrasonography. BMJ 306:1030

5. Brosens I, Campo R, Gordts S (1999) Office hydrolaparoscopy for the diagnosis of endometriosis and tubal infertility. Curr Opin Obstet Gynaecol 11:371

6. Gordts S, Campo R, Brosens I (2000) Office transvaginal hydrolaparoscopy for early diagnosis of pelvic endometriosis and adhesions. J Am Assoc Gynecol Laparosc 7:45
7. Moore ML, Cohen M (2001) Diagnostic and operative transvaginal hydrolaparoscopy for infertility and pelvic pain. J Am Assoc Gynecol Laparosc 8:393

8. Kikuchi A, Kozuma S, Sakamaki K, Saito M, Marumo G, Yasugi T, Taketani Y (2002) Fractal tumor growth of ovarian cancer: sonographic evaluation. Gynecol Oncol 87:295

9. Fathala MF (1971) Incessant ovulation - a factor in ovarian neoplasia. Lancet ii: 163

10. Cassagrande JT, Louie EW, Pike MC, Roy S, Henderson BE (1979) Incessant ovulation and ovarian cancer. Lancet ii:170

11. Leeper K, Garcia R, Swisher E, Goff B, Greer B, Paley P (2002) Pathologic findings in prophylactic oophorectomy specimens in high-risk women. Gynecol Oncol 87:52

12. Brosens I, Gordts S, Campo R (2001) Transvaginal hydrolaparoscopy but not standard laparoscopy reveals subtle endometriotic adhesions of the ovary. Fertil Steril 75:1009 